\title{
RADB: Random Access with Differentiated Barring for Latency-Constrained Applications in NB-IoT Network
}

\author{
Yiming Miao $\mathbb{D}$, ${ }^{1}$ Yuanwen Tian, ${ }^{1}$ Jingjing Cheng $\mathbb{D},{ }^{2,3}$ \\ M. Shamim Hossain $(1),{ }^{4}$ and Ahmed Ghoneim ${ }^{4,5}$ \\ ${ }^{1}$ School of Computer Science and Technology, Huazhong University of Science and Technology, Wuhan 430074, China \\ ${ }^{2}$ School of Automation, Huazhong University of Science and Technology, Wuhan 430074, China \\ ${ }^{3}$ Graduate School of System Informatics, Kobe University, Kobe 657-8501, Japan \\ ${ }^{4}$ Department of Software Engineering (SWE), College of Computer and Information Sciences (CCIS), King Saud University, \\ Riyadh 11543, Saudi Arabia \\ ${ }^{5}$ Computer Science Department, College of Science, Menoufia University, Menoufia 32721, Egypt
}

Correspondence should be addressed to Jingjing Cheng; chengjj@hust.edu.cn

Received 10 September 2017; Revised 12 November 2017; Accepted 27 November 2017; Published 10 January 2018

Academic Editor: Huimin Lu

Copyright (C) 2018 Yiming Miao et al. This is an open access article distributed under the Creative Commons Attribution License, which permits unrestricted use, distribution, and reproduction in any medium, provided the original work is properly cited.

With the development of LPWA (Low Power Wide Area) technology, the emerging NB-IoT (Narrowband Internet of Things) technology is becoming popular with wide area and low-data-rate services. In order to achieve objectives such as huge amount of connection and wide area coverage within NB-IoT, the problem of network congestion generated by random access of numerous devices should be solved. In this paper, we first introduce the background of NB-IoT and investigate the research on random access optimization algorithm. Then we summarize relevant features of NB-IoT uplink and narrowband physical random access channel and design random access with differentiated barring (RADB), which can improve the insufficiency of traditional dynamic access class barring method. At last, the algorithms proposed in this paper are realized with established NB-IoT model using OPNET Modeler platform, and simulations are conducted. The simulation results show that RADB is able to effectively solve preamble request conflict generated by random access of numerous devices and preferentially provide efficient and reliable random access for latency-sensitive devices.

\section{Introduction}

With the increase of low-data-rate and low power services $[1,2]$, research on LPWAN communication technology develops correspondingly [3]. Based on whether the spectrum is licensed, LPWAN communication technologies [4] are divided into the following types: type 1 includes technologies that run in unlicensed frequency band, such as Lora and Sigfox. Mostly, these technologies are nonstandard and customized, so that safety cannot be guaranteed. Type 2 includes technologies that run in licensed frequency band, including mature technologies such as $2 \mathrm{G} / 3 \mathrm{G}$ cellular communication technologies (GSM, CDMA, and WCDMA) and developing ones such as LTE and its evolution technology that are gradually deployed and applied at present which support terminals in various categories [5]. Basically, standards of these technologies are defined by international organizations for standard such as 3GPP (mainly formulates standards related to GSM, WCDMA, LTE, and its evolution technology) and 3GPP2 (mainly formulates standards related to CDMA).

NB-IoT is a kind of mass LPWA (Low Power Wide Area) technology put forth by 3GPP for application scenes with objectives of sensing and data acquisition (such as smart electric meter and environment monitoring [6]), characterized by advantages such as huge amount of connections, ultralow power consumption, wide area coverage, and mutual triggering between signaling $[7,8]$ and data [9-11]. In the meantime, it has support of excellent communication networks [12], such as cognitive vehicular networks [13] and cooperative communication networks [14].

In NB-IoT network, if a user equipment has access to the base station and sends service request, preamble request 
transmitted in NPRACH (narrowband physical random access channel) should be considered first, that is, random access procedure. However, when many users request the same preamble, preamble conflict occurs. What is worse, if there are too many preamble conflicts in the network where huge amounts of users request NPRACH resources, network congestion would be caused inevitably. At that moment, huge amount of failures in random access and long-term latency in network would take place. Therefore, an optimized model for random access is extremely urgent in order to improve QoS of network and QoE of user [15].

In allusion to problem of network congestion caused by access of huge amount of devices [16] in M2M network [17], 3GPP determines the following alternatives: (1) access class barring schemes; (2) separate RACH resources for MTC; (3) dynamic allocation of RACH resources; (4) MTC specific backoff scheme; (5) slotted access; (6) pull based scheme. However, solutions mentioned above have not taken the aspect of latency in random access into consideration and thus cannot provide efficient and reliable random access for latency-sensitive devices, like application scene in [18-20]. Therefore, RADB is put forth in this paper to solve problems mentioned above.

The remainder of this paper is organized as follows. Section 2 provides some related research work. Section 3 introduces the NPRACH (narrowband physical random access channel) features of NB-IoT, including random access concept and traditional dynamic access class barring method. Section 4 shows the proposed RADB and envisioned NBIoT architecture. Section 5 provides simulation setup and discusses experimental results. Finally, Section 6 concludes this paper.

\section{Related Work}

3GPP explicitly points out that it is necessary to give the theoretical computing model for uplink access latency when NB-IoT undertakes periodical and sudden MAR services [21]. Uplink access latency is composed of latencies in system synchronization, broadcast information reading, random access, resource allocation, data transmission and feedback response, and so on [7]. Among these latencies, some are deterministic processing latency, some are latencies related to signal detection, and others are random access latencies related to business activity [22]. Most projects of current research focus on computing of mean value and variance for random access latency; there are few projects of research that focus on probability density function (PDF) [23-25] of random access latency [26-28]. With quantity of waiting users and channel busy/idle as state variables, the moment generating function (MGF) for PDF of random access latency is deduced based on Markov process in [25, 29, 30]. But the problem of high computation complexity remains; it is even unsolvable when the quantity of users is too large. Reference [31] deduced PDF for random access latency on premise that time between arrivals and backoff obeys negative exponential distribution. References [23, 32] deduced PDF for random access latency on premise that retransmission times are fixed value or they obey geometric distribution. In research projects mentioned above, uniform distribution, exponential distribution, geometric distribution, and backoff mechanism are involved, but limitation of maximum retransmission times is taken into consideration only in [26, 33], which does not comply with actual protocol. The assumption that business models follow homogeneous Poisson or Bernoulli process is difficult to extend to application scenes of NBIoT. In combination with 3GPP beta type business model, approximate form of PDF for random access latency, is given in [34] by estimating the maximum retransmission times for terminals with successful access through mean value of latency. In [35], the lower bound for random access latency is deduced through approximate beta distribution of piecewise linear function, but the effect of maximum retransmission times is not taken into consideration. In short, the theoretical computing model for random access latency has not been completely solved up to now, as well as the simplest Poisson business model and uniform backoff mechanism. Therefore, the research of statistic characteristics for random access latency in NB-IoT in any random access strength (two scenes $\mathrm{C}$ restricted $\mathrm{PDCCH}$ and unrestricted PDCCH C are taken into consideration, resp.) grows wide attention; not only should mean value and variance be included, but also its PDF and corresponding MGF should be deduced to perfect random access latency analysis theory for NB-IoT.

\section{Narrowband Physical Random Access Channel Features}

The transmission bandwidth for uplink of NB-IoT system is $180 \mathrm{kHz}$, supporting two kinds of subcarrier spacing: $3.75 \mathrm{kHz}$ and $15 \mathrm{kHz}$. As for scenes with enhanced coverage, the subcarrier spacing of $3.75 \mathrm{kHz}$ may provide larger system capacity when compared with subcarrier spacing of $15 \mathrm{kHz}$. However, in the scenes with internal operation mode, subcarrier spacing of $15 \mathrm{kHz}$ has better compatibility with LTE, compared with subcarrier spacing of $3.75 \mathrm{kHz}$.

NB-IoT also reduces types of uplink physical channel, and some uplink physical channels are redesigned. Specifically, NB-IoT redesigns NPRACH and does not support PUCCH (Physical Uplink Control Channel).

3.1. NPRACH Features. Because the bandwidth of traditional LTE physical random access channel (PRACH) is $1.08 \mathrm{MHz}$, which exceeds restriction on bandwidth of $180 \mathrm{kHz}$ for uplink of NB-IoT, random access channel is redesigned and named as NPRACH. An NPRACH preamble is composed of four symbol groups, and each symbol group is composed of one cyclic prefix (CP) and five symbols. The CP with length of $66.67 \mathrm{~s}$ (Format 0 ) is suitable for cell with radius of $10 \mathrm{~km}$, and the CP with length of $266.7 \mathrm{~s}$ (Format 1 ) is suitable for cell with radius of $40 \mathrm{~km}$; thus the objective of coverage gain is achieved. The value of each symbol is fixed to 1 , and modulation is conducted at subcarrier spacing of $3.75 \mathrm{kHz}$ (with symbol duration of $266.67 \mathrm{~s}$ ). Thereinto, the frequency modulation index for each symbol group is different. However, the waveform of NPRACH preamble follows single-tone frequency hopping. Figure 1 shows a case of NPRACH frequency hopping [36]. In order to support 


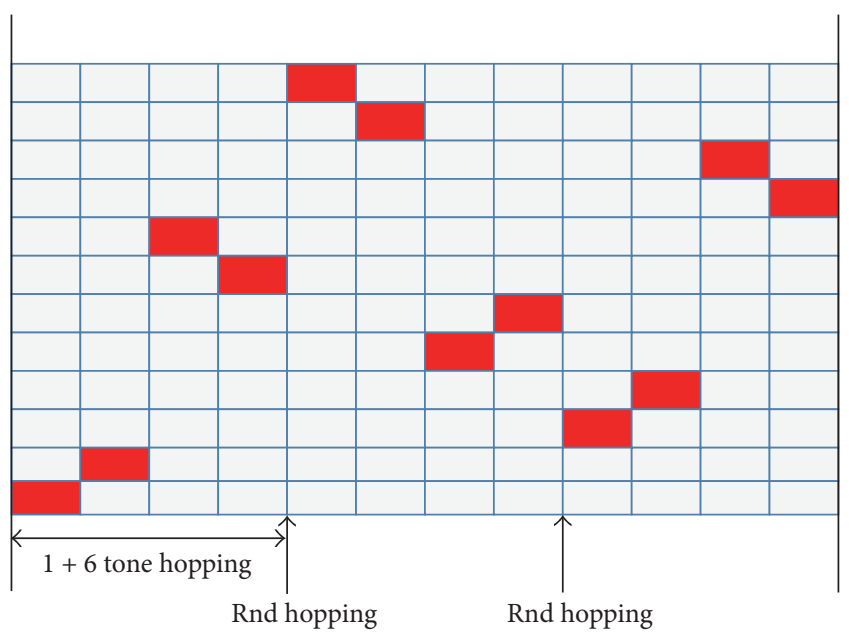

FIGURE 1: NPRACH frequency hopping.

coverage gain, the repeated use of one NPRACH preamble will be permitted for as many as 128 times.

3.2. Random Access. In allusion to requirements of coverage enhancement, random access based on coverage level is adopted by NB-IoT system. The terminal will judge the current coverage level based on signal strength measured, and appropriate random access resource shall be chosen based on corresponding coverage level to launch random access. In order to meet requirements on data transmission under different coverage levels, different times of repetition, transmission cycles, and so on will be allocated to each coverage level by base station. For example, the terminal under poor coverage level needs to adopt more times of repetition to guarantee correct transmission of data, while large transmission cycle may be allocated in order to prevent terminals under poor coverage level from occupying too many system resources.

Effective access control mechanism is required to guarantee and control access of terminal and preferential access of some abnormal data due to the huge amount of IoT terminals. As for access control mechanism of NB-IoT system, the EAB mechanism of LTE system (SIB14) and backoff mechanism of random access procedure are used for reference. Also, MIB$\mathrm{NB}$ broadcasts indication of access control to reduce power consumption of SIB14-NB that tries to read at terminal.

In NB-IoT, random access is used in many aspects such as initial access during establishing wireless link [37] and scheduling request [38]. A main objective of random access is to realize synchronization of uplink, which plays a vital role in maintaining orthogonality of uplink. Similar to random access mechanism of LTE, competition-based random access procedure of NB-IoT includes the following four steps: (1) user equipment (UE) sends a random access preamble; (2) a random access response (including timing advance command and uplink resources scheduling) will be transmitted by network for use of UE in the third step; (3) UE broadcasts its identity label in the network with scheduled resources; (4) contention-resolution message is transmitted by network to solve conflict caused when multiple UE pieces send the same random access preamble in the first step.

In order to better serve UE pieces under different coverage levels and with different degrees of path loss, up to 3 kinds of different NPRACH resources will be allocated in a cell by NB-IoT network. In each kind of allocation, each basic random access preamble has a given duplicate value for repeated use. UE will measure its signal receiving power at downlink to estimate its coverage level and adopt NPRACH resources allocated by network to send random access preamble for the estimated coverage level. In order to deploy NB-IoT network in different scenes, flexible allocation of NPRACH resources under time-frequency resource grid is allowed by NB-IoT; the specific parameters are as follows:

(i) Time domain: NPRACH resource has periodicity referring to the start time of NPRACH resource in a period of time.

(ii) Frequency domain: it includes frequency distribution (determined by subcarrier migration) and quantity of subcarrier.

In early field test and deployment of NB-IoT, some UE pieces do not support multitone transmission. Therefore, before transmission scheduling for uplink, the network should know multitone transmission capacity of UE. In addition, in the first step of random access procedure, a UE should express information on whether it supports multitone transmission, so that transmission scheduling for uplink can be realized by the network in the third stage of random access procedure. To be specific, network divides NPRACH subcarriers into two nonoverlapping sets by their frequency domain. In the third step of random access procedure, UE may choose one of the two sets to send its signal of random access preamble and thus to express whether it supports multitone transmission.

Consequently, UE determines its coverage level by measuring signal receiving power at downlink. After reading system information allocated by NPRACH resources, UE is able to conduct NPRACH resource allocation and to set retransmission times required by estimating its coverage level and transmission power of random access preamble. Then, UE is able to continuously and repeatedly transmit basic single-tone random access preamble within a period of NPRACH resources.

However, continuous retransmission of single-tone random access preamble in a single cycle may cause preamble request conflict. With a large amount of conflicts increasing the request and response delay time (i.e., random access latency would be longer), the network will fall into congestion inevitably. Access request of huge amount of devices will bring great challenge to wireless access capacity of access network while the main focus is on overload problem in a cell as for congestion in access network. For example, assuming that large amounts of devices access a cell simultaneously, the conflict probability of access channel in that cell will increase rapidly, and severe paralysis will be caused if control is not available in time. 


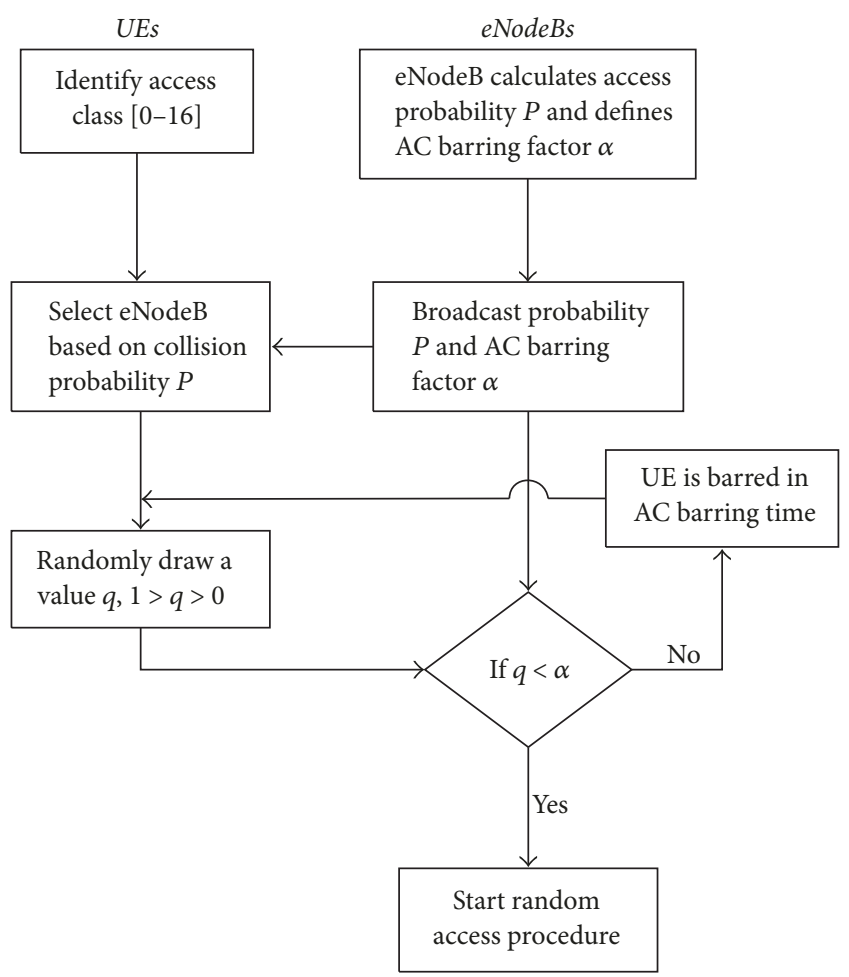

FIgURE 2: Access class barring schemes.

3.3. Access Class Barring Schemes. The solution to solve network congestion caused by large amount of access requests in cellular machine-to-machine (M2M) network is put forth in [39], that is, dynamic access class barring (ACB) method. Figure 2 shows the ACB method, including base station selection and load balance strategy. Main steps are as follows:

(i) Step 1: eNodeB evaluates the conflict probability $P$ of random access based on the arrival rate and transmission rate of data package. Access class barring parameter $\alpha$ depends on PRACH condition (total preamble number and current requests number for preamble).

(ii) Step 2: every eNodeB periodically broadcasts conflict probability $P$ and AC barring parameter $\alpha$.

(iii) Step 3: UE chooses the corresponding eNB when the maximum access success probability is achieved and conducts connection and communication to the eNB.

(iv) Step 4: each UE will generate a random number $q$ (1 > $q>0$ ), and start/prohibit random access procedure following ACB mechanism; that is, UE is able to successfully start random access procedure when and only when $q<\alpha$.

\section{Random Access with Differentiated Barring and Network Architecture}

4.1. Random Access with Differentiated Barring. In RADB put forth in this paper, conflict probability, random number generation mechanism, AC barring parameter $\alpha$, and access recognition algorithm of traditional ACB scheme are defined specifically; then corresponding improvement is made.

Devices in NB-IoT network are divided into Class $A$ and Class $B$. Class $A$ stands for latency-sensitive devices (that long for low data transmission latency), while Class $B$ stands for non-latency-sensitive devices (that may tolerate longer data transmission latency). NPRACH period $T$ is divided into $t$ time slots and random number $q_{(t)}$ of UE pieces in each time slot is defined in formula (1), where $t=0,1,2, \ldots, T$.

$$
\begin{gathered}
q_{A(t)}, \quad t=0,1,2, \ldots, T ; \\
q_{B(t)} \in(0,1), \quad t=0,1,2, \ldots, T .
\end{gathered}
$$

In allusion to preamble defined in eNodeB, we assume the sum is $S$, request number of current preamble is $x$, and channel conflict probability is $P$, as shown in the following formula:

$$
P=1-\left(1-\frac{1}{S}\right)^{(x-1)}
$$

If $P=1-(1-1 / S)^{(x-1)}<0.1$, it is held that the channel conflict probability is low; that is, success rate for random access is high. In this case, it is assumed that the maximum value of $x$ is $X$, and $X$ stands for the maximum request number of preamble when success rate for random access is guaranteed. Let number of Class $A$ devices be $N_{A}$ and number of Class $B$ devices be $N_{B}$; then the total number of devices $N=N_{A}+N_{B}$, and the number of devices with successful access $N_{s}=N_{A}+N_{B}$. Therefore, dynamic AC barring parameter $\alpha$ may be set as per the following formula:

$$
\alpha= \begin{cases}1, & N<X ; \\ \frac{X-N_{A}}{N_{B}}, & N>X>N_{A} ; \\ 0, & N_{A}>X .\end{cases}
$$

Because $q_{A(t)}$ is always equal to 0 (remaining constant while $t$ changes), $q_{A(t)}$ is always less than or equal to $\alpha$; in other words, devices in Class $A$ have the right to start random access procedure preferentially. Only when redundancy in sum of preambles (sum of preambles is more than number of devices in Class $A$ that request preamble) appears, could devices in Class $B$ have the chance to access NB-IoT eNodeB. The pseudocode of RADB Scheme is shown as Algorithm 1.

4.2. NB-IoT Network Architecture. In accordance with previous research [40], the architecture of NB-IoT network established based on OPNET Modeler platform in this paper is as shown in Figure 3, mainly including 5 parts: NB-IoT terminal, NB-IoT access network, NB-IoT core network, NBIoT cloud platform, and vertical industry center $[41,42]$. In order to embody coverage gain attribute of NB-IoT network and three corresponding NPRACH resource configuration options, the whole network is divided into 3 areas from long range to short range, and 3 MCSs (MCS 9, MCS 20, and MCS28) are selected, respectively, as modulation and coding strategy for each area based on MCS index table given in [40]; MCS index ID is made as area ID. In each area, NB-IoT 


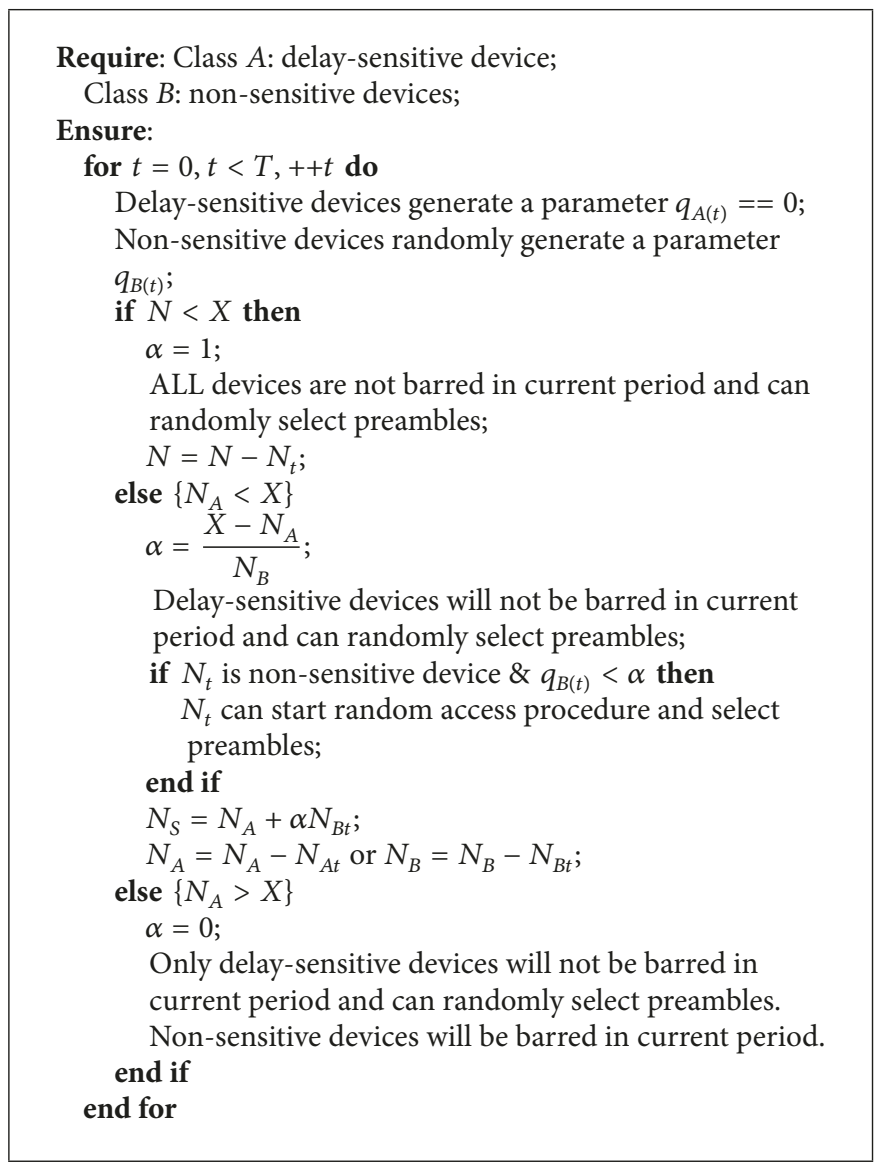

Algorithm 1: Random access with differentiated barring.

terminals (UE pieces, standing for devices carried by different mobile users) in different numbers are deployed; local ID in an area is made as identity label of these devices in that area. All user devices in the network send random access request to corresponding NB-IoT base station (improved LTE base station, eNodeB); whether an equipment could successfully enter random access procedure is determined through RADB.

As shown in Figure 4, when the synchronous relationship between an NB-IoT terminal and base station is not established, that terminal must send random access request before it could access network, that is, from idle state to connected state. At that moment, it is difficult for limited system information and channel information to guarantee reliability of transmission with closed loop random access control; therefore, Algorithm 1 is adopted to improve and set up random access process model in physical layer in this paper.

\section{Simulation and Analysis}

Table 1 lists the values of important parameters considered in the simulations [40]. These values were selected to reflect real-world implementations of NB-IoT network and based on our previous research [40]. The simulations were run multiple times and the presented results are an average of these runs.
Firstly, comparison is made between access success rate of NB-IoT network with different quantities of equipment under RADB and that of NB-IoT network under traditional access class barring scheme when barring parameter $\alpha$ is 0.2 or 0.8 , as shown in Figure 5 . When the quantity of equipment is less than 150 , the performance of ACB scheme with $\alpha$ of 0.2 is still fine. However, with increase in quantity of equipment, the RADB that dynamically adjusts barring parameter shows strong controlling force over random access; it effectively makes sure that latency-sensitive equipment could successfully access network to the largest degree.

Figure 6 shows the comparative results for access latency generated with different random access models in NB-IoT network with 350 mobile devices; the random access models include RADB and traditional access class barring scheme with barring parameter $\alpha$ equal to $0.2,0.4,0.6$, and 0.8 . It is shown that the latency for ACB scheme with barring parameter $\alpha$ of 0.8 is the longest (latency for ACB scheme with barring parameter $\alpha$ of 0.6 is the second longest), which indicates that large amounts of devices access network at that time leading to the increasing probability of channel conflict and frequent network congestion. However, though the latency for ACB scheme with barring parameter $\alpha$ of 0.2 and 0.4 is short, the quantity of devices that can successfully access network at that time is too small; therefore instability of network is caused. As for RADB, with setting of dynamic 
TABLE 1: Simulation parameters.

\begin{tabular}{|c|c|c|}
\hline Element & Attribute & Value \\
\hline \multirow{6}{*}{ EPS } & QoS class identifier & 1 (GBR) \\
\hline & Allocation retention priority & 2 \\
\hline & Uplink guaranteed bit rate & $32 \mathrm{Kbps}$ \\
\hline & Downlink guaranteed bit rate & $96 \mathrm{Kbps}$ \\
\hline & Uplink maximum bit rate & $32 \mathrm{Kbps}$ \\
\hline & Downlink maximum bit rate & $384 \mathrm{Kbps}$ \\
\hline \multirow{8}{*}{ Physical layer profiles } & UL SC-FDMA channel & \\
\hline & Base frequency & $1920 \mathrm{MHz}$ \\
\hline & Bandwidth & $0.2 / 3 / 5 / 10 / 15 / 20 \mathrm{MHz}$ \\
\hline & Cyclic prefix type & 7 symbols per slot \\
\hline & DL OFDMA channel & \\
\hline & Base frequency & $2110 \mathrm{MHz}$ \\
\hline & Bandwidth & $0.2 / 3 / 5 / 10 / 15 / 20 \mathrm{MHz}$ \\
\hline & Cyclic prefix type & 7 symbols per slot \\
\hline \multirow{2}{*}{ eNodeB } & Failure/recovery specification time & 200 seconds \\
\hline & Barring parameter $\alpha$ of ACB & $0.2 / 0.4 / 0.6 / 0.8$ \\
\hline \multirow{5}{*}{ UE pieces } & Battery capacity & 5 \\
\hline & Maximum transmission power & $10 \mathrm{~mW}$ \\
\hline & Number & 50,500 per 50 \\
\hline & Modulation and coding scheme index & $9 / 20 / 28$ \\
\hline & Operating power & $100 \mathrm{~mW}$ \\
\hline
\end{tabular}

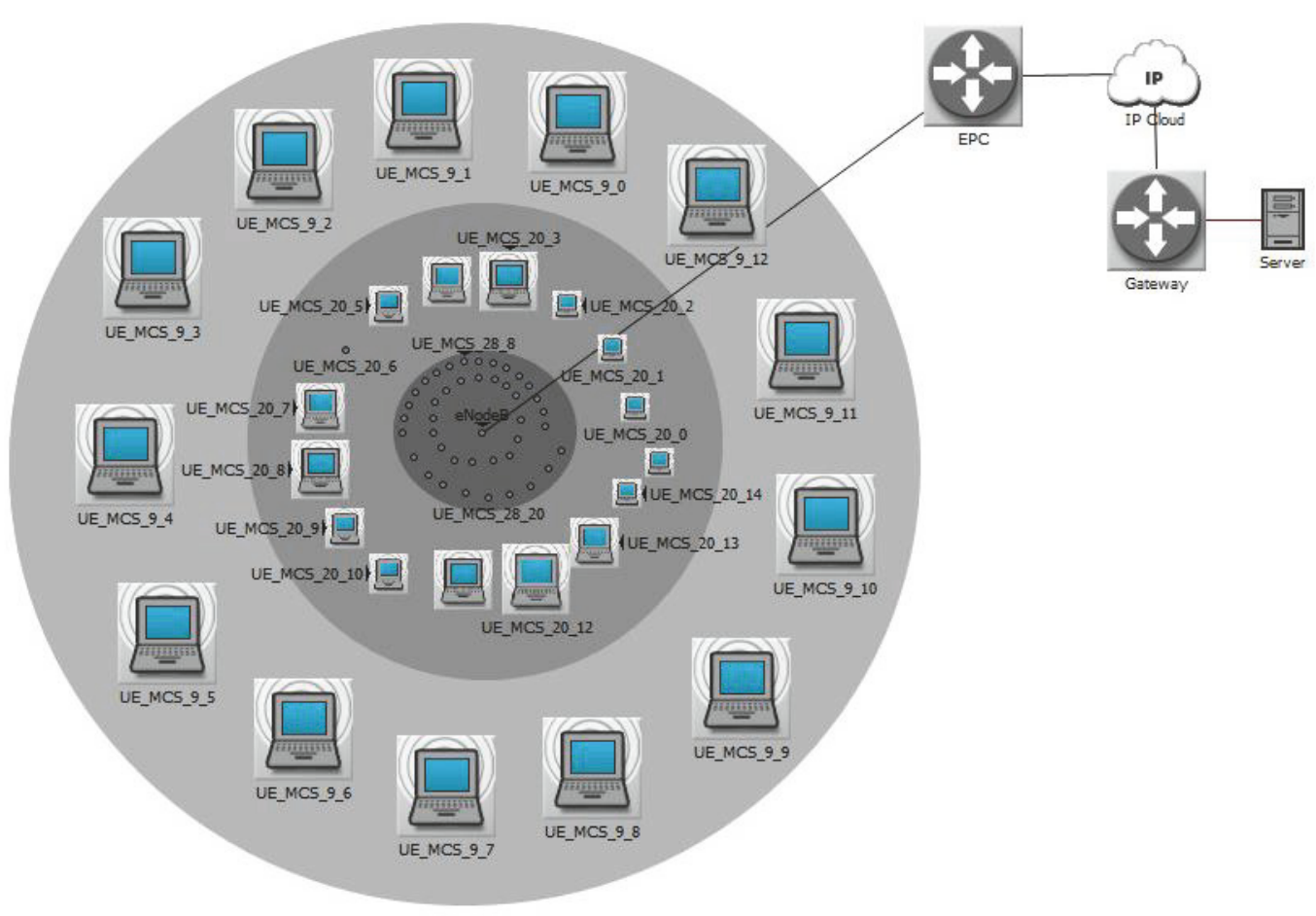

MCS 28

MCS 20

MCS 9

FIGURE 3: NB-IoT network architecture. 


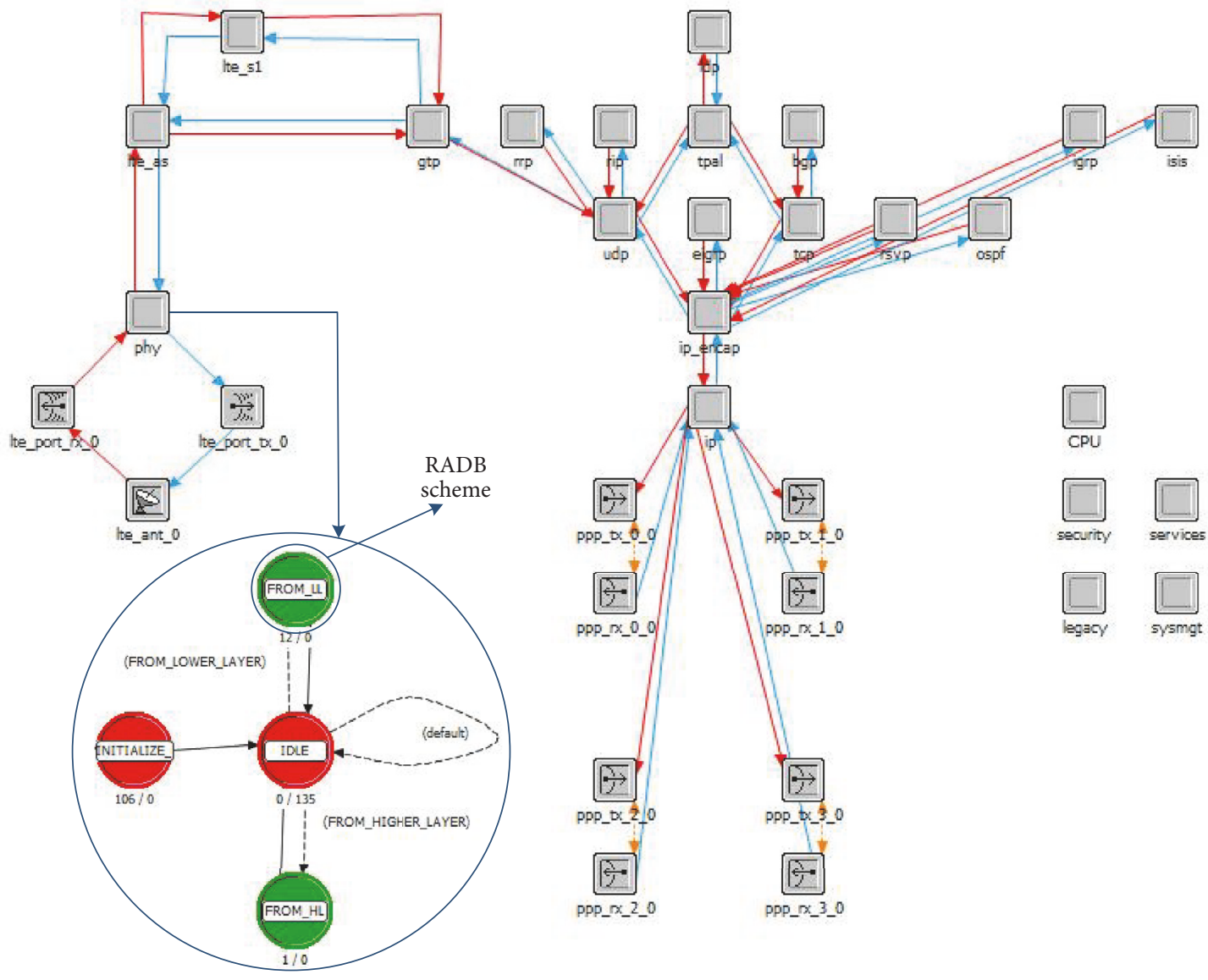

FIGURE 4: RADB process model.

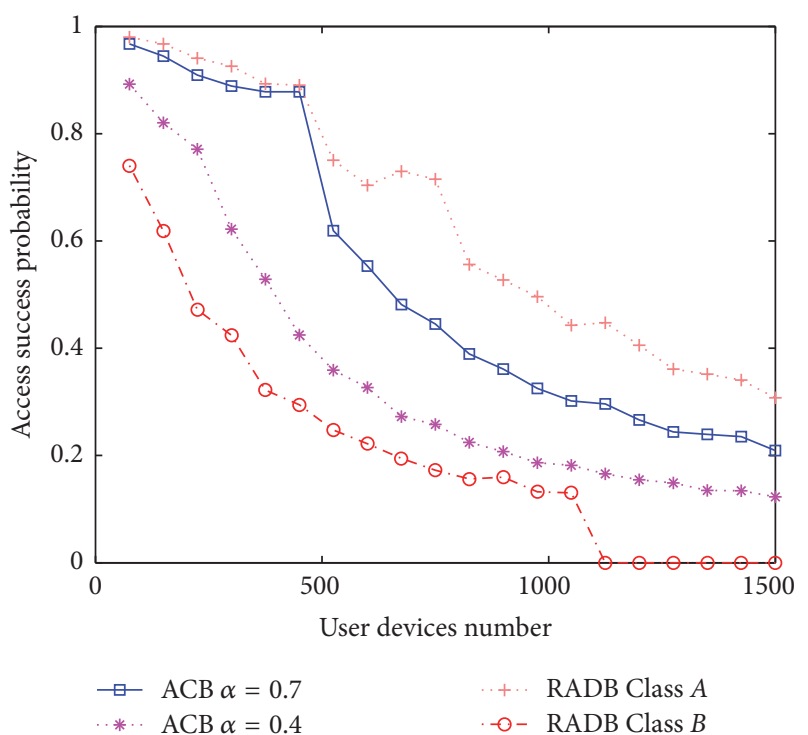

Figure 5: Access success probability.

barring parameter, network could enter stable state earlier; thus network latency is controlled effectively.

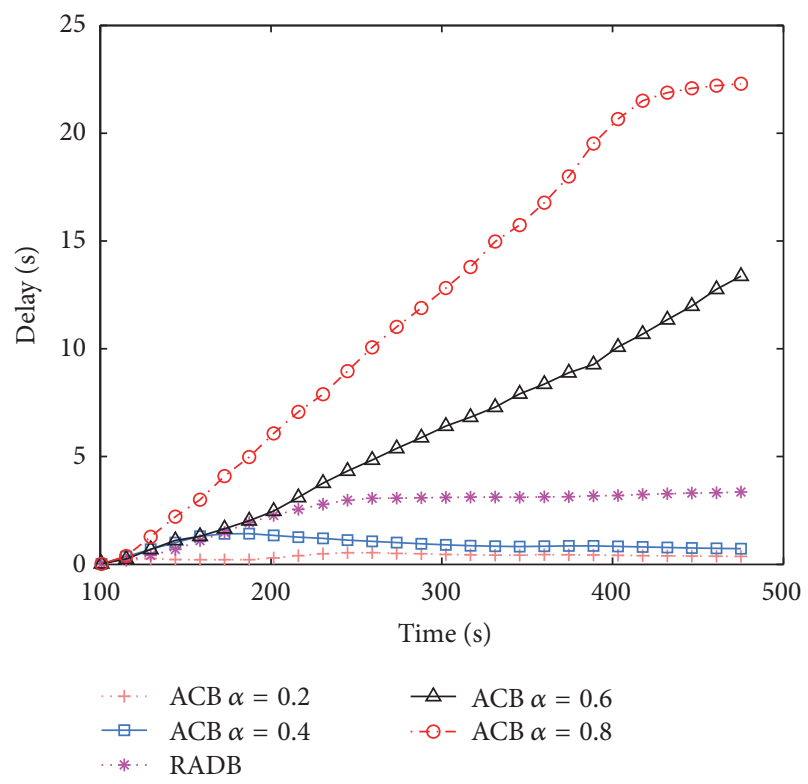

Figure 6: Access delay.

Figure 7 shows the comparative results for network load generated with different random access models in NB-IoT 


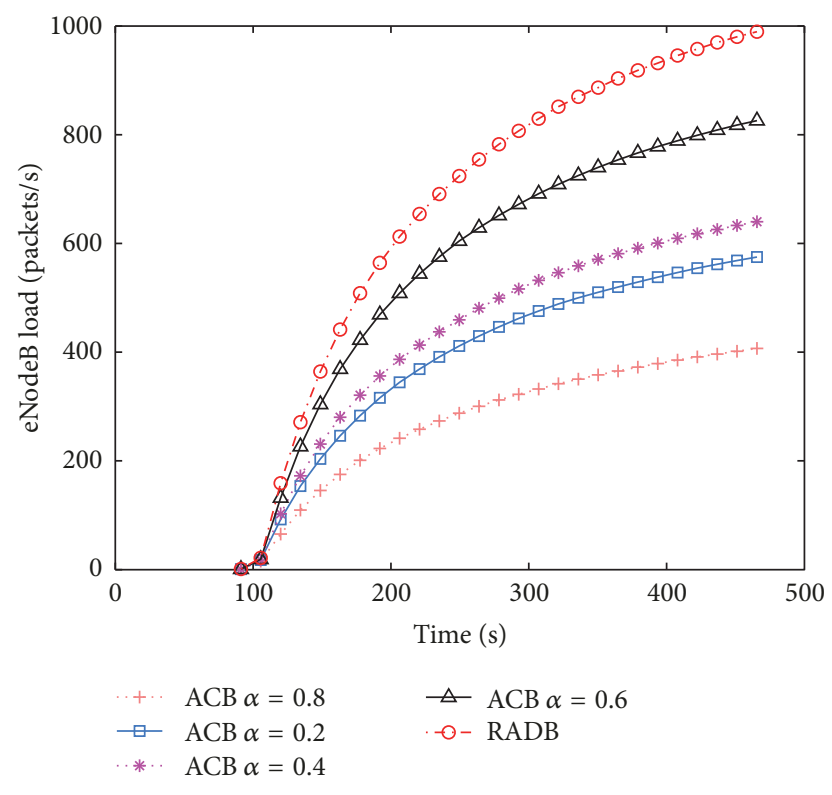

Figure 7: eNodeB load.

network with 350 mobile devices. The throughput (loading capacity) of a system is closely related to the consumption of CPU by request, peripheral interface, IO, and so on. If the consumption of CPU by single request is higher, the response rate of peripheral interface and $\mathrm{IO}$ is slower and the throughput of the system is lower. This situation is the opposite when the CPU consumption is very low. It can be seen that the response rate of base station is very slow when $\mathrm{ACB}$ scheme $\alpha$ is equal to 0.8 due to long access latency; therefore, the network throughput is very low. When $\mathrm{ACB}$ scheme $\alpha$ is equal to 0.2 , though access latency is short, devices that access network are fewer, so the network throughput is not high. However, as for RADB put forth in this paper, because network latency is controlled, the requirements of latency-sensitive devices on network are met; thus network throughput is guaranteed.

\section{Conclusion}

In this paper, the background of NB-IoT is introduced and worldwide research related to optimized algorithms for random access is investigated. Then, characteristics related to NB-IoT uplink and narrowband physical random access channel are summarized, improvement is made in allusion to insufficiency of traditional dynamic access class barring method, and RADB is designed. Furthermore, the algorithms put forth in this paper are realized with established NBIoT model, and simulation experiment is conducted. The results of simulation experiment show that RADB is able to effectively solve preamble request conflict generated by random access of numerous devices and to preferentially provide efficient and reliable random access for latencysensitive devices.

Nevertheless, problems of channel resource distribution and resource utilization rate are not taken into consideration in algorithm put forth in this paper. In subsequent research, we will continue to study equipment access algorithms with high energy efficiency and low load and improve existing models, thus providing theoretical and experimental bases for future large-scale deployment of NB-IoT network in the real world. Also, how to use Big Data techniques [43] to support the NB-IoT based services is still interesting but challenging.

\section{Conflicts of Interest}

The authors declare that they have no conflicts of interest.

\section{Acknowledgments}

The authors extend their appreciation to the Deanship of Scientific Research at King Saud University, Riyadh, Saudi Arabia, for funding this work through the research group Project no. RGP-229.

\section{References}

[1] X. Hou, Y. Li, M. Chen, D. Wu, D. Jin, and S. Chen, "Vehicular fog computing: a viewpoint of vehicles as the infrastructures," IEEE Transactions on Vehicular Technology, vol. 65, no. 6, pp. 3860-3873, 2016.

[2] F. Xu, Y. Li, H. Wang, P. Zhang, and D. Jin, "Understanding Mobile Traffic Patterns of Large Scale Cellular Towers in Urban Environment," IEEE/ACM Transactions on Networking, vol. 25, no. 2, pp. 1147-1161, 2017.

[3] M. Chen, Y. Miao, Y. Hao, and K. Hwang, "Narrow Band Internet of Things," IEEE Access, vol. 5, pp. 20557-20577, 2017.

[4] X. Xiong, K. Zheng, R. Xu, W. Xiang, and P. Chatzimisios, "Low power wide area machine-to-machine networks: Key techniques and prototype," IEEE Communications Magazine, vol. 53, no. 9, pp. 64-71, 2015.

[5] M. Chen, Y. Hao, L. Hu, K. Huang, and V. Lau, "Green and Mobility-aware Caching in 5G Networks," IEEE Transactions on Wireless Communications, vol. 16, no. 12, pp. 8347-8361, 2017.

[6] K. Lin, M. Chen, J. Deng, M. Hassan, and G. Fortino, "Enhanced fingerprinting and trajectory prediction for IoT localization in smart buildings," IEEE Transactions on Automation Science and Engineering, vol. 13, no. 3, pp. 1294-1307, 2016.

[7] 3GPP TR 45.820, "Cellular system support for ultra-low complexity and low throughput cellular internet of things," 2015.

[8] 3GPP TS 36.211, "E-UTRA Physical channels and modulationChap.10 Narrowband IoT," 2016.

[9] C.-C. Tseng, H.-C. Wang, F.-C. Kuo, K.-C. Ting, H.-H. Chen, and G.-Y. Chen, "Delay and power consumption in LTE/LTEA DRX mechanism with mixed short and long cycles," IEEE Transactions on Vehicular Technology, vol. 65, no. 3, pp. 17211734, 2015.

[10] R. Cheng, A. Deng, and F. Meng, Study of NB-IoT Planning Objectives And Planning Roles, China Mobile Group Design Institute Co., 2016.

[11] K. Lin, J. Luo, L. Hu, M. S. Hossain, and A. Ghoneim, "Localization based on social big data analysis in the vehicular networks," IEEE Transactions on Industrial Informatics, vol. 13, no. 4, pp. 1932-1940, 2017.

[12] K. He, J. Chen, R. Du, Q. Wu, G. Xue, and X. Zhang, "DeyPoS: Deduplicatable Dynamic Proof of Storage for Multi-User 
Environments," IEEE Transactions on Computers, vol. 65, no. 12, pp. 3631-3645, 2016.

[13] D. Tian, J. Zhou, Z. Sheng, and V. C. M. Leung, "Robust Energy-Efficient MIMO Transmission for Cognitive Vehicular Networks," IEEE Transactions on Vehicular Technology, vol. 65, no. 6, pp. 3845-3859, 2016.

[14] D. Tian, J. Zhou, Z. Sheng, M. Chen, Q. Ni, and V. C. Leung, "Self-Organized Relay Selection for Cooperative Transmission in Vehicular Ad-Hoc Networks," IEEE Transactions on Vehicular Technology, vol. 66, no. 10, pp. 9534-9549, 2017.

[15] F. Xu, Z. Tu, Y. Li, P. Zhang, X. Fu, and D. Jin, "Trajectory Recovery From Ash: User Privacy Is NOT Preserved in Aggregated Mobility Data," in Proceedings of the 26th International Conference on World Wide Web, pp. 1241-1250, 2017.

[16] J. Chen, K. He, Q. Yuan, G. Xue, R. Du, and L. Wang, "Batch identification game model for invalid signatures in wireless mobile networks," IEEE Transactions on Mobile Computing, vol. 16, no. 6, pp. 1530-1543, 2017.

[17] M. Chen, J. Wan, S. Gonzalez, X. Liao, and V. C. M. Leung, "A survey of recent developments in home M2M networks," IEEE Communications Surveys \& Tutorials, vol. 16, no. 1, pp. 98-114, 2014.

[18] M. Chen, Y. Hao, M. Qiu, J. Song, D. Wu, and I. Humar, "Mobility-aware caching and computation offloading in 5G ultra-dense cellular networks," Sensors, vol. 16, no. 7, pp. 974987, 2016.

[19] M. Chen, J. Yang, X. Zhu, X. Wang, M. Liu, and J. Song, "Smart home 2.0: innovative smart home system powered by botanical IoT and emotion detection," Mobile Networks and Applications, vol. 22, pp. 1159-1169, 2017.

[20] M. Chen, Y. Hao, K. Hwang, L. Wang, and L. Wang, "Disease Prediction by Machine Learning Over Big Data From Healthcare Communities," IEEE Access, vol. 5, pp. 8869-8879, 2017.

[21] A. Laya, L. Alonso, and J. Alonso-Zarate, "Is the random access channel of LTE and LTE-A suitable for M2M communications? A survey of alternatives," IEEE Communications Surveys \& Tutorials, vol. 16, no. 1, pp. 4-16, 2014.

[22] K. Lin, J. Song, J. Luo, W. Ji, M. Shamim Hossain, and A. Ghoneim, "Green Video Transmission in the Mobile Cloud Networks," IEEE Transactions on Circuits and Systems for Video Technology, vol. 27, no. 1, pp. 159-169, 2017.

[23] Y. Yang and T.-S. P. Yum, "Delay Distributions of Slotted ALOHA and CSMA," IEEE Transactions on Communications, vol. 51, no. 11, pp. 1846-1857, 2003.

[24] C.-H. Wei, P.-C. Lin, and R.-G. Cheng, "Comment on 'an efficient random access scheme for OFDMA systems with implicit message transmission"', IEEE Transactions on Wireless Communications, vol. 12, no. 1, pp. 414-415, 2013.

[25] A. Mutairi, S. Roy, and G. Hwang, "Delay analysis of OFDMAaloha," IEEE Transactions on Wireless Communications, vol. 12, no. 1, pp. 89-99, 2013.

[26] F. A. Tobagi, "Distributions of packet delay and interdeparture time in slotted ALOHA and Carrier Sense Multiple Access," Journal of the ACM, vol. 29, no. 4, pp. 907-927, 1982.

[27] J.-B. Seo and V. C. M. Leung, "Design and analysis of backoff algorithms for random access channels in UMTS-LTE and IEEE 802.16 systems," IEEE Transactions on Vehicular Technology, vol. 60, no. 8, pp. 3975-3989, 2011.

[28] K. Zheng, Z. Yang, K. Zhang, P. Chatzimisios, K. Yang, and W. Xiang, "Big data-driven optimization for mobile networks toward 5G," IEEE Network, vol. 30, no. 1, pp. 44-51, 2016.
[29] L. Dai, "Stability and delay analysis of buffered aloha networks," IEEE Transactions on Wireless Communications, vol. 11, no. 8, pp. 2707-2719, 2012.

[30] K. Zheng, F. Liu, L. Lei, C. Lin, and Y. Jiang, "Stochastic performance analysis of a wireless finite-state Markov channel," IEEE Transactions on Wireless Communications, vol. 12, no. 2, pp. 782793, 2013.

[31] M. E. Rivero-Angeles, D. Lara-Rodríguez, and F. A. Cruz-Pérez, "Access delay analysis of adaptive traffic load - Type protocols for S-ALOHA and CSMA in EDGE," in Proceedings of the 2003 IEEE Wireless Communications and Networking Conference: The Dawn of Pervasive Communication, WCNC 2003, vol. 3, pp. 1722-1727, March 2003.

[32] M. E. Rivero-Angeles, D. Lara-Rodriguez, and F. A. Cruz-Perez, "Gaussian approximations for the probability mass function of the access delay for different backoff policies in S-ALOHA," IEEE Communications Letters, vol. 10, no. 10, pp. 731-733, 2006.

[33] R. R. Tyagi, F. Aurzada, K.-D. Lee, and M. Reisslein, “Connection Establishment in LTE-A networks: Justification of poisson process modeling," IEEE Systems Journal, vol. 99, pp. 1-12, 2015.

[34] C.-H. Wei, G. Bianchi, and R.-G. Cheng, "Modeling and analysis of random access channels with bursty arrivals in OFDMA wireless networks," IEEE Transactions on Wireless Communications, vol. 14, no. 4, pp. 1940-1953, 2015.

[35] M. Koseoglu, "Lower Bounds on the LTE-A Average Random Access Delay under Massive M2M Arrivals," IEEE Transactions on Communications, vol. 64, no. 5, pp. 2104-2115, 2016.

[36] Y. E. Wang, X. Lin, A. Adhikary et al., "A Primer on 3GPP Narrowband Internet of Things," IEEE Communications Magazine, vol. 55, no. 3, pp. 117-123, 2017.

[37] S.-S. Kim, S. McLoone, J.-H. Byeon, S. Lee, and H. Liu, "Cognitively Inspired Artificial Bee Colony Clustering for Cognitive Wireless Sensor Networks," Cognitive Computation, vol. 9, no. 2, pp. 207-224, 2017.

[38] H. Liu, A. Abraham, V. Snášel, and S. McLoone, "Swarm scheduling approaches for work-flow applications with security constraints in distributed data-intensive computing environments," Information Sciences, vol. 192, pp. 228-243, 2012.

[39] L. Ferdouse and A. Anpalagan, "A dynamic access class barring scheme to balance massive access requests among base stations over the cellular M2M networks," in Proceedings of the 26th IEEE Annual International Symposium on Personal, Indoor, and Mobile Radio Communications, PIMRC 2015, pp. 1283-1288, September 2015.

[40] Y. Miao, W. Li, D. Tian, M. S. Hossain, and M. F. Alhamid, "Narrow Band Internet of Things: Simulation and Modelling," IEEE Internet of Things Journal, vol. PP, no. 99, pp. 1-1, 2017.

[41] Y. Li and M. Chen, "Software-defined network function virtualization: a survey," IEEE Access, vol. 3, pp. 2542-2553, 2015.

[42] Y. Li, F. Zheng, M. Chen, and D. Jin, "A unified control and optimization framework for dynamical service chaining in software-defined NFV system," IEEE Wireless Communications Magazine, vol. 22, no. 6, pp. 15-23, 2015.

[43] X. Wang, Y. Zhang, V. C. M. Leung, N. Guizani, and T. Jiang, "D2D big data: content deliveries over wireless device-to-device sharing in realistic large scale mobile networks," IEEE Wireless Commun, vol. 25, no. 1, pp. 1-10, 2018. 


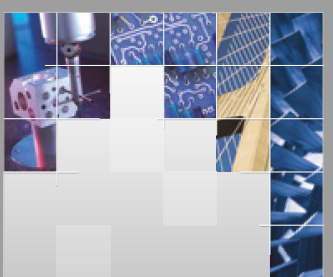

\section{Enfincering}
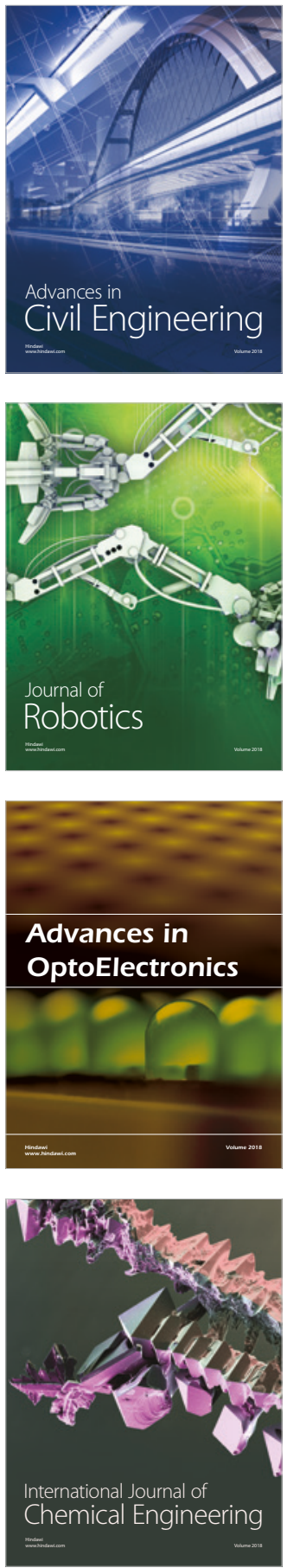

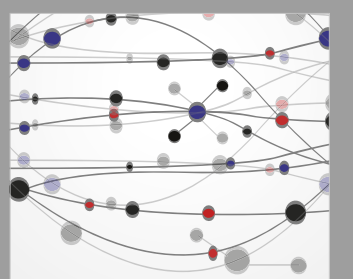

\section{Rotating \\ Machinery}

The Scientific World Journal

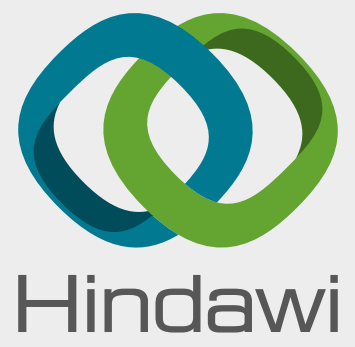

Submit your manuscripts at

www.hindawi.com
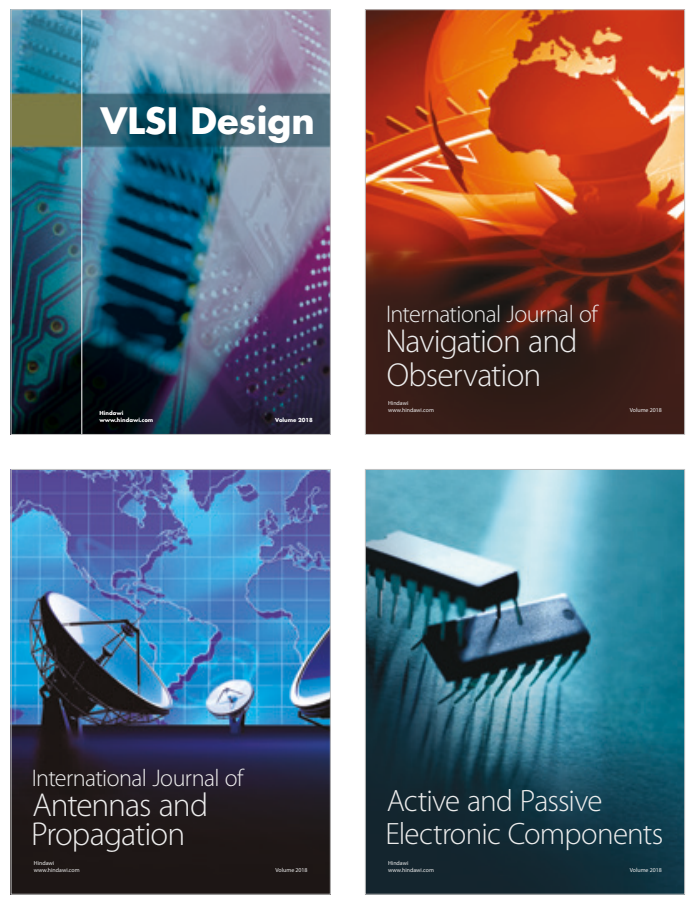
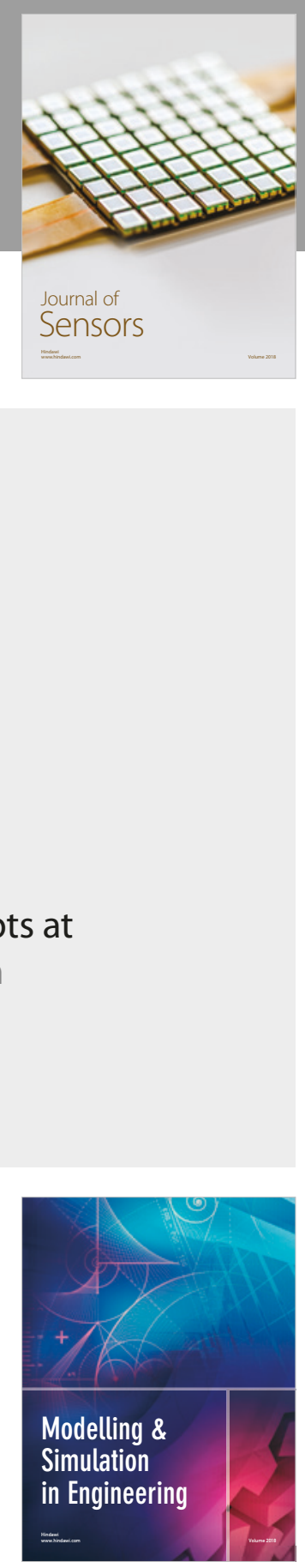

\section{Advances \\ Multimedia}
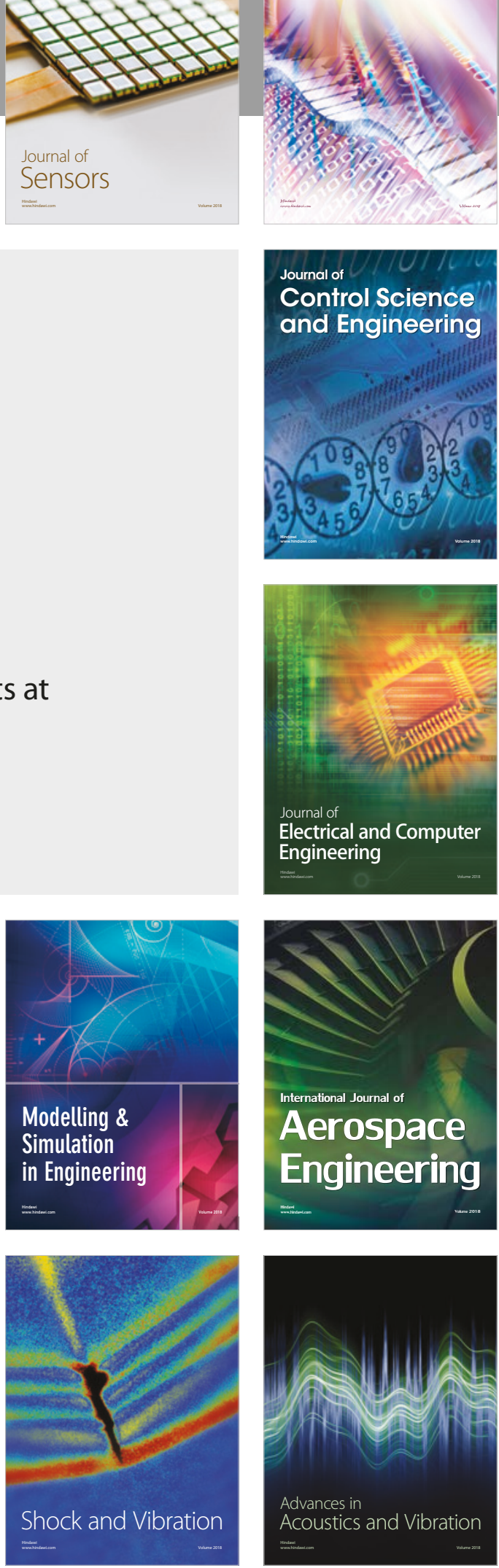